power equal to a fourth of that of dextrose-in other words, it requires 40 milligrammes of creatinine to produce the amount of reducing action that is effected by ten milligrammes of dextrose. Under this reckoning the reducing effect on Fehling's solution produced by the one to two grammes, or about this, of creatinine conjectured to be eliminated per diem would count for exceedingly little in relation to the total reducing action exerted by normal urine, instead of occupying a position of significance, as there is a disposition to be assumed it does by some observers.

Calculating in the same way as was done for uric acid, the apportionment of the reducing power of normal urine to be assigned to creatinine comes out as follows : if 1.5 grammes of creatinine and 1500 cubic centimetres of urine be taken as representing the elimination amounts for the 24 hours the relation of creatinine to urine will stand at 1 per 1000 . 40 milligrammesiof creatinine, as shown above, may be taken as having the same reducing power as 10 milligrammes of dextrose. The reducing power therefore to be reckoned for the one gramme of creatinine is equivalent to $\frac{1 \times 10}{40}$ $=0.25$ per 1000 expressed as dextrose. The mean total reducing power for normal urine was found to be 2.79 per 1000 expressed as dextrose, and, from the data given, 0.25 per 1000 of this, or practically one-eleventh, constitutes the proportion to be assigned to creatinine.

(To be continued.)

\section{A CASE OF CEREBRO-SPINAL MENINGITIS DURING PREGNANCY.}

BY J. D. WILLIAMSON, M.D., M.CH., M.A.O. R.U.I., PHYSICIAN FOR DISEASES OF CHILDREN AND OPSTETRICS, ULSTER HOSPIT.AL FOR CHILDREN AND WOMEN, BELFAST.

THE following are a few notes of a case of cerebro-spinal meningitis during pregnancy. It is interesting from more than one point of view : the age and condition of the patient, the onset of labour (whilst declaring that she did not know what was the matter with her and denying she was pregnant); the sudden onset of cerebro-spinal meningitis during labour, its rapid conrse, leaving no time for any very active treatment of service ; the look of the patient ; the character of the petechial and herpetic eruptions, without head symptoms ; and the persistent vomiting, which in this case, at the time being associated with labour, did not demand the same amount of attention that in other circumstances it would have received.

The patient was in a neighbouring town up to Christmas, 1896, when she came home to Belfast to a street in a working-class locality. She was aged 13 years and 9 months; her mother gave the age in evidence at the coroner's court as 14 years on May 23rd, 1907. She was admitted on March 5th, 1907, to the maternity department of the Ulster Hospital for Children and Women as being in labour. The patient had always been a strong, healthy girl, well developed, and had never menstruated. The history of the case as reported to me was that on March 25th, about 2 A.M., the patient was seized with violent pains in the lower part of the back and abdomen, accompanied with vomiting; the pains were not continuous but paroxysmal; she became so ill that a medical man was sent for about 4 A.M. He prescribed medicine and advised certain measures to give her some relief; later he (the medical man) returned accompanied by another medical man, and on consultation both agreed that the patient was in labour. This the patient denied, protesting against anything of the kind. On telling her mother what was the matter the latter expressed her astonishment at anything of the sort as she never thought such a thing possible owing to her age and appearance. The patient absolutely refused to give any information regarding her condition to anyone. At 12 noon the patient was admitted to the Ulster Hospital. There was then a temperature of $101^{\circ} \mathrm{F}$. and the pulse was 100 , full, and bounding. There were strong aterine contractions at intervals of about ten minutes. There was no vomiting. There was slight mucoid diarrhoea, the cheeks were slightly cyanosed, and there was herpes about the nostrils and the mouth. No rash at this time was present; the tongue was furred but moist, and the pupils reacted normally. She had a wild and frightened appearance, was quite conscious, and complained of no pain in the head.

On vaginal examination the passage was moist and dilatable, the os was very slightly dilated, the presentation being a vertex but very high up in the pelvis. The patient remained in much the same condition until 2 P.M., when the pains became almost continuous, breathing was very rapid and distressed, the cheeks and lips were more deeply cyanosed, and the pulse was rapid and irregular. I was at this stage summoned by telephone to see the patient who was reported as being in the maternity ward and in labour. Almost immediately I received a second message to say that the patient was in a state of collapse and to come at once. I replied to give a hypodermic injection of strychnine and I would go over immediately, which I did and found the patient in bed lying on her right side, the legs flexed on the thighs, and the thighs on the abdomen; the forearm was flexed on the arm and both the arms were lying on the front of the chest; she was conscious, the pupils were slightly dilated and equal in size, the face was cyanosed, the breathing was rapid and difficult, the pulse was quick and irregular, and she was to all appearance in labour. She was nervous and frightened looking but did everything she was asked to do.

I got her turned over on to the left side and made a vaginal examination with some difficulty; the passage had the feeling as if contracting on the finger; the rugæ of the vagina were well defined. With some difficulty I reached the os, which was slightly dilated, admitting the tip of my finger. I was able to make out the presentation a vertex. With the left hand on the abdomen I could feel the uterus hard and contracting; her attitude and appearance did not harmonise in my opinion with the stage of labour and I felt that there might be something the matter in her chest. On examining the chest I arrived at the opinion that there was well-marked congestion of both lungs, the heart was acting rapidly and irregularly, and I was unable to make out any valvular lesion. No urine could be procured by catheter. Neither the face nor the extremities were oedematous. I also noted that she was very sensitive to the touch (hyperæsthetic). I got her turned on the back in order to examine the abdomen to learn if the child were still alive. The breasts were well developed, secondary areolæ were well marked, and there was a knotted feeling, and on pressure fluid exuded through the nipples. On stethoscoping over the uterus the fotal heart sounds were heard very indistinctly and I observed some dark spots of a peculiar shape and appearance over the lower part of the abdomen and the thighs, which on investigation I learned had developed since her admission to hospital. The spots were dark in colour, irregular in shape and size, some flat, others raised like a small blister, and on pressure the colour did not disappear. These with her look and condition suggested cerebro-spinal meningitis. She, however, very quickly got worse though still remaining perfectly conscious, complain-ing only of the uterine contractions and pain in the back. There was no pain in the head but great pain and difficulty in swallowing, together with heavy breathing, death supervening about 3.50 P.M.

The patient's condition was so serious when I first saw her that I felt no obstetrical interference would prove of any service or obviate a fatal result to either mother or child rapid dilatation of the os and removal of the fotus might have been attempted but had I endeavoured to do any thing of the kind death would have followed even more quickly and question, could I have saved the child had it been alive? Removal of the foetus by Crsarean section might have been adopted but I doubt very much if the child conld have been saved.

In conjunction with Dr. J. Martin I made a post-mortem examination that night in the public mortuary. The body was stout, well built, well developed like a woman of small stature, and well nourished. There were no marks of any kind on the body other than the dark spotted rash (as described) over the abdomen and the thighs. Rigidity was well marked. On opening the skull the membranes of the brain were congested and the blood-vessels were full; also there was a greater quantity of serous fluid than ordinary in the cavity of the cranium. The brain was otherwise natural in appearance; there was more serous fluid than normal in the ventricles, and the internal blood-vessels were congested. The spinal cord was not examined; as the evidence of cerebro-spinal meningitis was so manifest we 
thought it unnecessary. The pleura was congested and the pleural cavity contained more serous fluid than natural. The lungs were dark and markedly congested. With regard to the pericardium the cavity contained more serous fluid than normal. The heart was slightly enlarged and the valves were healthy. Ante-mortem clot was present. As to the abdomen, the peritoneum was congested and spotted all over with black spots of various sizes and different shapes. There was more serous fluid than normal in the pelvic cavity. The uterus extended up close to the xiphoid cartilage of the sternum and corresponded with a uterus at full term. The peritoneum over it was congested and contained dark spots. On opening the uterus a full-term male child was removed; it was a well-developed, healthy-looking infant ; the skin was healthy and without any spots or marks of any kind. The placenta, membranes, and cord were normal in size and appearance. The mesenteric glands were congested, which has been shown lately in Germany to be important from a diagnostic point of view, a view confirmed by Pro fessor W. St.C. Symmers of Belfast. The liver was enlarged and full of blood. The gall-bladder was full of bile and the coating of the bladder was covered with dark spots. The spleen was enlarged and full of blood. The kidneys and suprarenal capsules were congested. The pancreas was congested. The stomach contained a little food with some dark grumous matter which was evidently the medicine prescribed that morning. There were dark spots over the mucous surface, which was also congested. The small and large intestines were congested and almost empty; Peyer's patches were congested and the walls of the intestines were spotted. The bladder was empty and on opening it the mucous surface was covered over the whole surface with small dark spots. As this case occurred in the earlier stage of the epidemic I regret bacteriological examinations were not made.

\section{THE TREATMENT OF TRYPANOSOMIASIS. ${ }^{1}$}

By A. NIERENSTEIN, PH.D.,

OF THE LIVERPOOL SCHOOL OF TROPICAL MEDICINE.

THE therapeutics of the present time for trypanosomiasis can be divided into the following.

A. Arsenic compounds.-1. Arsenious acid. 2. Fowler's solution.

$B$. Colours.-1. Colouring matters belonging to the diazo group, Ehrlich's trypanred and Mesnil's afrodol blue and afrodol violet. 2. Colouring matters belonging to the triphenyl-methan group, Ehrlich's malachite green and fuchsin.

C. Atoxyl, which being an organic compound of aniline and arsenious acid cannot be considered as merely "an arsenic compound," an expression recently used in the lay press.

The introduction of an acid radicle into an amido nucleus generally changes its basic character and of course, on the other hand, lessens the acidity. The combined effect of aniline and arsenic is therefore the resultant of these two factors: amido group and arsenious acid. We have been able to demonstrate experimentally that either the amount of aniline generally given in the form of atoxyl or the arsenious acid contained in that drug when administered alone will kill an animal within a few hours after the injection.

$D$. Combined treatment. - 1 . Combination of arsenious or acid atoxyl with colouring matters, as trypanred suggested by Thomas and Breinl, Laveran and Mesnil, and others. 2. Atoxyl and strychnine-van Campenhout. 3. Atoxyl and sublimate. The last-named treatment has recently been worked ont in the Liverpool School of Tropical Medicine and the results obtained on rats are very promising.

We who have been working in the Liverpool School of Tropical Medicine found that in infected rats (Trypanosoma Brucei), one part of which were treated with atoxyl alone and the remainder with atoxyl followed by mercury, the first lot generally had relapses in from 16 to 25 days, while a great number of the animals treated with atoxyl and mercury are still alive, some after a period of nine months. These results are certainly encouraging. In our treatment we used, at the beginning, to give high doses of atoxyl in order to drive out the parasites entirely and it was found that if sufficient

1 A paper read before the Society of Tropical Medicine and Hygiene on July 10th, 1907. atoxyl were administered the parasites (Trypanosoma Brucei) would disappear in rats for from 16 to 25 days. A dis. appearance of the parasites for a similar period was also observed in guinea-pigs and rabbits. Mercury was then given and the treatment stopped. In the bigger animals this double treatment should be continued for some time.

With regard to sleeping sickness we would like to suggest as a rontine treatment that one cubic centimetre of a 20 per cent. solution of atoxyl should be given daily for one week, followed by one cubic centimetre of a 1 per cent. solution of sublimate four times. This treatment should be repeated and continued for an extended period and, of course, may require occasional modification. It would perhaps be possible to give mercury in the form of pills. The theory of the action of mercury in this treatment has been discussed in a paper published in the Annals of Tropical Medicine and Parasitology of the Liverpool School of Tropical Medicine, Vol. I., No. 2.

It has been found that the toxic effect of atoxyl can be diminished by acetylating the atoxyl. Such an acetylated atoxyl is an antipyrine which contains arsenious acid. Acetylated atoxyl has been shown to be of use in mice by Ehrlich and in dogs by our experiments. We found, as a rale, that when atoxyl was given in a sufficient quantity to drive out the parasites (nagana) in dogs the host was also killed, but using acetylated atoxyl we have been more or less successful. An interesting fact, pointed out by Ehrlich, is that the parasites can get used to a drug and become resistant. It is therefore advisable to alternate various trypanocidal agents in the treatment as much as possible, that is, to give atoxyl, some other trypanosome-killing drug, and mercury; for example, atoxyl, fuchsin, or some other colouring matter by the mouth, and sublimate.

It has usually been recommended to give atoxyl in high doses at the beginning. Whether or not this is of benefit is a question, as the atoxyl apparently accumulates in the body and the toxic effect appears after a time. In laboratory experiments we have frequently noticed that even after the treatment had been stopped for a week toxic effects appeared and this could only be due to the fact that atoxyl had accumulated in the body and the arsenic had been slowly broken up. We would like to refer to one experiment; a rat was treated about nine months ago with atoxyl and is still showing toxic effects.

We think it necessary to point out that the administration of atoxyl should be given a very careful trial but that extremely high doses should be avoided as much as possible. The action of atoxyl seems to be very much on the nervous system, as neuritis is frequently caused by the administration of this drug. Blindness has also been observed after the use of atoxyl and the question therefore arises whether it would not be well to counteract somewhat the effect of atoxyl by making it less operative on the brain and nerves. This might be done by introducing radicles which are more fixed to the blood and less to the nervous system.

We think it necessary to draw attention to experiments which we have made in connexion with the reappearance of trypanosomes after treatment with atoxyl. We found that strains obtained from animals which had had relapses were sometimes much more virulent. Our nagana strain, used for work generally, killed a rat in from five to seven days. This strain was followed up, commencing on Jan. 14th, until May 5 th by subinoculation from one rat to another; four drops of blood were always used. In none of these rats was any sudden increase of virulence noticed, but in experiments with the strain obtained from animals in which the trypanosomes had reappeared it was found that the experimental animal was killed in from three to five days. Out of 17 rats used for these experiments 11 showed a remarkable increase of virulence. Similar work done with Trypanosoma dimorphon showed the same increase of viralence. Our strain used to kill a rat in from 15 to 17 days and we found that in four out of six strains obtained through relapses after treatment by atoxyl the virulence obtained was from 11 to nine days. We have recently obtained through atoxyl a Trypanosoma dimorphon strain which kills a rat in seven days. Similar work with Trypanosoma Gambiense is now in progress.

It was our intention not to publish the results of this work until we had satisfied ourselves that Trypanosoma Gambiense behaves in a similar way, but since it is intended to commence atoxyl treatment on a large scale in Uganda we feel that a warning in this direction should be given.

These observations emphasise the necessity for careful 